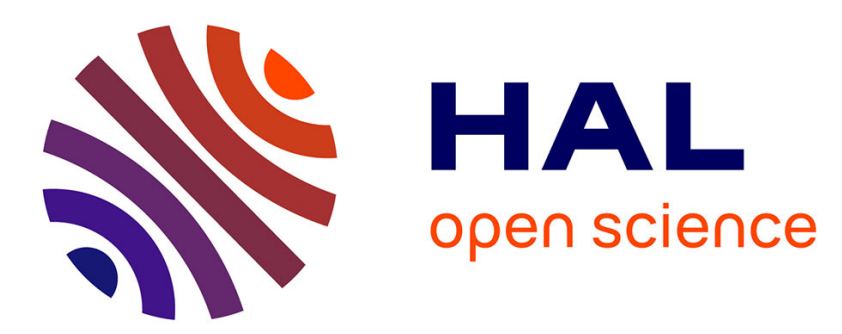

\title{
On the Dynamic Properties of Rigid-Link Flexible-Joint Parallel Manipulators in the Presence of Type 2 Singularities
}

\author{
Sébastien Briot, Vigen Arakelian
}

\section{- To cite this version:}

Sébastien Briot, Vigen Arakelian. On the Dynamic Properties of Rigid-Link Flexible-Joint Parallel Manipulators in the Presence of Type 2 Singularities. Journal of Mechanisms and Robotics, 2010. hal-00473631

\section{HAL Id: hal-00473631 \\ https://hal.science/hal-00473631}

Submitted on 25 Jun 2019

HAL is a multi-disciplinary open access archive for the deposit and dissemination of scientific research documents, whether they are published or not. The documents may come from teaching and research institutions in France or abroad, or from public or private research centers.
L'archive ouverte pluridisciplinaire HAL, est destinée au dépôt et à la diffusion de documents scientifiques de niveau recherche, publiés ou non, émanant des établissements d'enseignement et de recherche français ou étrangers, des laboratoires publics ou privés. 


\author{
Sébastien Briot \\ Dpt. de Génie Mécanique et Au- \\ tomatique - L.G.C.G.M. EA3913 \\ Institut National des Sciences \\ Appliquées \\ Rennes, France \\ sbriot@yahoo.fr \\ Vigen Arakelian \\ vigen.arakelian@insa-rennes.fr
}

\section{On the Dynamic Properties of Rigid-Link Flexible-Joint Parallel Manipulators in the Presence of Type 2 Singu- larities}

\begin{abstract}
In our previous work [1], the dynamic properties of rigid-link parallel manipulators, in the presence of Type 2 singularities, have been studied. It was shown that any parallel manipulator can pass through the singular positions without perturbation of motion if the wrench applied on the end-effector by the legs and external efforts of the manipulator are orthogonal to the twist along the direction of the uncontrollable motion. This condition was obtained using symbolic approach based on the inverse dynamics and the study of the Lagrangian of a general rigid-link parallel manipulator. It was validated by experimental tests carried out on the prototype of a four-degrees-of-freedom parallel manipulator. However, it is known that flexibility of the mechanism may not always been neglected. Indeed, joint flexibility is the main source contributing to overall manipulator flexibility and it leads to the trajectory distortion. Therefore, in this paper, the condition for passing through a Type 2 singularity of parallel manipulators with flexible joints is studied. The suggested technique is illustrated by the example of a $5 R$ parallel manipulator with flexible joints. It is shown that passing through singularity is possible if the twelfth order polynomial trajectory planning is applied. The obtained results are validated by numerical simulations carried out using the ADAMS software.
\end{abstract}

\section{Introduction}

Over the past decades, with the large development of parallel manipulators, more attention has been paid to their kinematic, kinetostatic and dynamic properties, and in particular, to their singularities. Several papers deal with singularity analysis of parallel manipulators [2-14]. Most of them present the analysis of singular configurations from a kinematic point of view [2-8]. Algebraically, a singularity analysis is based on the degeneracy of Jacobian matrices of the mechanical structure, or of the system of reciprocal screws (wrenches) applied to the platform by the legs. However, it is also known that, when parallel manipulators have Type 2 singularities [2], they lose their stiffness and their quality of motion transmission, and as a result, their payload capability. Therefore, the singularity zones in the workspace of manipulators may be analyzed not only in terms of kinematic criterions, from the theoretically perfect model of manipulators, but also in terms of kinetostatic approaches [9-14].

Moreover, while it is demonstrated using the kinetostatic approach that, when subjected to Type 2 singularities parallel manipulator lock up, it has been shown experimentally that, via optimal dynamic control of manipulators, it is possible to pass through these singular zones. Thus, it is evident that singular configurations should also be examined in terms of dynamic aspects.

The further study of singularity in parallel manipulators has revealed an interesting problem that concerns the path planning of parallel manipulators under the presence of singular positions, i.e. the motion feasibility in the neighborhood of singularities. In this case the dynamic conditions can be considered in the design process. One of the most evident solutions for the stable motion generation in the neighborhood of singularities is to use redundant sensors and actuators [15]-[18]. However, it is an expensive solution to the problem because of the additional actuators and the complicated control of the manipulator caused by actuation redundancy. Another approach concerns with motion planning to pass through singularity [19]-[25], i.e. a parallel manipulator may track a path through singular poses if its velocity and acceleration are properly constrained. This is a promising way for the solution of this problem. However, only a few research papers on this approach have addressed the path planning for obtaining a good tracking performance. But they have not adequately addressed the physical interpretation of dynamic aspects.

In our recent work [1], optimal force generation in parallel manipulators for passing through the singular positions has been studied. It was shown that any parallel manipulator can pass through the singular positions without perturbation of motion if the wrench applied on the end-effector by the legs and external efforts of the manipulator are orthogonal to the twist along the direction of the uncontrollable motion. This paper was concerned with the study of rigid-link parallel manipulators without any flexibility. However, it should be noted that several factors may bring a loss of rigidity in parallel manipulators (elasticity of links, clearance in joints, etc.). But their contribution can be considerably reduced for a properly designed and constrained mechanical system. However, even for the most optimum design of manipu- 
lator one main source of flexibility remains and it cannot be easily reduced: it is the flexibility in actuated joints, due to the use of Harmonic Drive ${ }^{\circledR}$ systems.

Therefore, in the present work the dynamic condition for passing through the singular positions is defined in general for parallel manipulators taking into account the elasticity in the actuated joints.

The paper is organized as follows. The next section presents theoretical aspects of the examined problem. As in our previous work, using the Lagrangian formulation, the condition of force distribution is defined, that allows the passing of any parallel manipulator through the Type 2 singular positions. In section 3, the suggested solution is illustrated via $5 \mathrm{R}$ planar parallel manipulator. In section 4 , the conclusions are given.

\section{Optimal dynamic conditions for passing through Type 2 singularity}

Let us consider a parallel manipulator of $m$ links, $n$ degrees of freedom and driven by $n$ actuators.

The general Lagrangian dynamic formulation for a manipulator with elasticity in actuated joints can be expressed as [26]:

$$
\begin{gathered}
\mathbf{0}=\frac{\mathrm{d}}{\mathrm{dt}}\left(\frac{\partial L}{\partial \dot{\mathbf{q}}_{d}}\right)-\frac{\partial L}{\partial \mathbf{q}_{d}}, \\
\boldsymbol{\tau}=\frac{\mathrm{d}}{\mathrm{dt}}\left(\frac{\partial L}{\partial \dot{\mathbf{q}}_{a}}\right)-\frac{\partial L}{\partial \mathbf{q}_{a}},
\end{gathered}
$$

where,

- $L$ is the Lagrangian of the manipulator; $L=T-V$, where $T$ is the kinetic energy and $V$ the potential energy due to gravitational forces, friction and elasticity;

- $\mathbf{q}_{a}=\left[q_{1}^{a}, q_{2}^{a}, \ldots, q_{n}^{a}\right]^{T}$ and $\dot{\mathbf{q}}_{a}=\left[\dot{q}_{1}^{a}, \dot{q}_{2}^{a}, \ldots, \dot{q}_{n}^{a}\right]^{T}$ represent the vectors of position and velocity of the actuators, respectively;

- $\mathbf{q}_{d}=\left[q_{1}^{d}, q_{2}^{d}, \ldots, q_{n}^{d}\right]^{T}$ and $\dot{\mathbf{q}}_{d}=\left[\dot{q}_{1}^{d}, \dot{q}_{2}^{d}, \ldots, \dot{q}_{n}^{d}\right]^{T}$ represent the vectors of position and velocity of controlled links, respectively, i.e. the position and velocity of the links that are controlled by the displacement of the actuators in which there are elasticity;

$-\tau$ is the vector of the actuators efforts.

However, for a parallel mechanism, the position (velocity, resp.) of the end-effector is a non-trivial function of the position (velocity, resp.) of the controlled links, therefore it is preferable to rewrite Eq. (1) using the Lagrange multipliers, as follows:

$$
\begin{gathered}
\mathbf{0}=\mathbf{W}_{\mathbf{b}}+\mathbf{B}^{T} \boldsymbol{\lambda}, \mathbf{W}_{\mathbf{b}}=\frac{\mathrm{d}}{\mathrm{dt}}\left(\frac{\partial L}{\partial \dot{\mathbf{q}}_{d}}\right)-\frac{\partial L}{\partial \mathbf{q}_{d}} \\
\boldsymbol{\tau}=\frac{\mathrm{d}}{\mathrm{dt}}\left(\frac{\partial L}{\partial \dot{\mathbf{q}}_{a}}\right)-\frac{\partial L}{\partial \mathbf{q}_{a}},
\end{gathered}
$$

where $\lambda$ is the Lagrange multipliers vector, which is related to the wrench applied on the platform by:

$$
\lambda=\mathbf{A}^{-T} \mathbf{W}_{\mathbf{p}}, \mathbf{W}_{\mathbf{p}}=\left(\frac{\mathrm{d}}{\mathrm{dt}}\left(\frac{\partial L}{\partial \mathbf{v}}\right)-\frac{\partial L}{\partial \mathbf{x}}\right)
$$

where,

- $\mathbf{A}$ and $\mathbf{B}$ are two matrices relating the vectors $\mathbf{v}$ and $\dot{\mathbf{q}}$ according to $\mathbf{A v}=\mathbf{B} \dot{\mathbf{q}}_{d}$. They can be found by differentiating the closure equations with respect to time;
- $\mathbf{x}=[x, y, z, \phi, \psi, \theta]^{T}$ and $\mathbf{v}=[\dot{x}, \dot{y}, \dot{z}, \dot{\phi}, \dot{\psi}, \dot{\theta}]^{T}$ are trajectory parameters and their derivatives, respectively; $x, y, z$ represent the position of the controlled point and $\phi, \psi$ and $\theta$ the rotation of the platform about three axes $\mathbf{a}_{\phi}, \mathbf{a}_{\psi}$ and $\mathbf{a}_{\theta}$;

- $\mathbf{W}_{\mathbf{p}}$ is the wrench applied on the platform by the legs and external forces [27] expressed along axes $\mathbf{a}_{\phi} \mathbf{a}_{\psi}$ and $\mathbf{a}_{\theta}$.

Expressing $\mathbf{W}_{\mathbf{p}}$ in the base frame, one can obtain:

$$
\begin{gathered}
\mathbf{0}=\mathbf{W}_{\mathbf{b}}+\mathbf{J}^{T} \mathbf{R}_{0} \mathbf{W}_{\mathbf{p}}, \\
\boldsymbol{\tau}=\frac{\mathrm{d}}{\mathrm{dt}}\left(\frac{\partial L}{\partial \dot{\mathbf{q}}_{a}}\right)-\frac{\partial L}{\partial \mathbf{q}_{a}},
\end{gathered}
$$

where $\mathbf{J}=\left({ }^{\mathbf{R}_{0}} \mathbf{A}\right)^{-1} \mathbf{B}$ is the Jacobian matrix between twist $\mathbf{t}$ of the platform (expressed in the base frame) and $\dot{\mathbf{q}}_{d},{ }^{\mathbf{R}_{0}} \mathbf{A}=\mathbf{A} \mathbf{D}$ is the expression of matrix $\mathbf{A}$ in the base frame, where $\mathbf{D}$ is a transformation matrix, of which expression is given in [28].

For any prescribed trajectory $\mathbf{x}(t)$, the values of vectors $\ddot{\mathbf{q}}_{d}$, $\dot{\mathbf{q}}_{d}$ and $\mathbf{q}_{d}$ can be found using the inverse kinematics. Thus, taking into account that the manipulator is not in a Type 1 singularity [2], the terms $\mathbf{W}_{\mathbf{b}}$ and ${ }^{\mathbf{R}_{0}} \mathbf{W}_{\mathbf{p}}$ can be computed. However, for a trajectory passing through a Type 2 singularity, the determinant of matrix $\mathbf{J}$ is indefinite. Numerically, the values of the efforts applied by the actuators become infinite. In practice, the manipulator either is locked in such a position of the end-effector or it can not follow the prescribed trajectory.

It is known that a Type 2 singularity appears when the determinant of matrix ${ }^{\mathbf{R}_{0}} \mathbf{A}$ vanishes, in other words, when at least two of its columns are linearly dependant [28]. So, one may obtain such a relationship:

$$
\sum_{j=1}^{6} \alpha_{j} \mathbf{A}_{j}=\mathbf{0}
$$

where $\mathbf{A}_{j}$ represents the $j$-th column of matrix ${ }^{\mathbf{R}_{0}} \mathbf{A}$ and $\alpha_{j}$ are coefficients, which in general can be functions of $q_{p}^{d}(p=1, \ldots$, $n)$. It should be noted that the vector $\mathbf{t}_{\mathbf{s}}=\left[\alpha_{1} \alpha_{2}, \ldots, \alpha_{6}\right]^{T}$ represents the direction of the uncontrollable motion of the platform in a Type 2 singularity.

By substituting (5) into (3), we obtain

$$
\mathbf{A}_{j}^{T} \boldsymbol{\lambda}=W_{j}, \mathrm{j}=1, \ldots, 6
$$

where $W_{j}$ is the $j$-th row of vector ${ }^{\mathbf{R}_{0}} \mathbf{W}_{\mathbf{p}}$.

Then, from (5) and (6) the following conditions are derived:

$$
\sum_{j=1}^{6}\left(\alpha_{j} \mathbf{A}_{j}^{T} \boldsymbol{\lambda}\right)=\sum_{j=1}^{6}\left(\alpha_{j} W_{j}\right)=0 .
$$

The right term of Eq. (7) corresponds to the scalar product of vectors $\mathbf{t}_{\mathbf{s}}$ and ${ }^{\mathbf{R}_{0}} \mathbf{W}_{\mathbf{p}}$.

Thus, in the presence of a Type 2 singularity, it is possible to satisfy conditions (7) if the wrench applied on the platform by the legs and external efforts ${ }^{R_{0}} W_{p}$ are orthogonal to the direction of the uncontrollable motion $\mathbf{t}_{\mathbf{s}}$. Otherwise, the dynamic model is not consistent. Obviously, in the presence of a Type 2 singularity, the displacement of the end-effector of the manipulator has to be planned to satisfy (7). Therefore, our task will be to 
achieve a trajectory which will allow the manipulator to pass trough the Type 2 singularities, i.e. which will allow the manipulator respecting condition (7).

In the dynamic model of the rigid-link flexible-joint manipulator [26], the efforts $\tau$ applied on the actuators may be expressed as follows:

$$
\boldsymbol{\tau}=\frac{\mathrm{d}}{\mathrm{dt}}\left(\frac{\partial L}{\partial \dot{\mathbf{q}}_{d}}\right)-\frac{\partial L}{\partial \mathbf{q}_{d}}=\mathbf{M}\left(\mathbf{q}_{d}\right) \ddot{\mathbf{q}}_{d}+\mathbf{C}\left(\mathbf{q}_{d}, \dot{\mathbf{q}}_{d}\right) \dot{\mathbf{q}}_{d}+\mathbf{D}\left(\mathbf{q}_{d}\right),
$$

This equation only depends on the acceleration, velocity and position of the actuators of the manipulator. Therefore, in order to avoid some discontinuity on the efforts $\tau$, the polynomial used for the trajectory planning should be at least of degree 5 (because initial and final positions are known, and the velocities and accelerations at the beginning and the end of the trajectory should be equal to 0 ). In our previous work [1], it was shown that the condition for passing through the Type 2 singular configurations added 3 supplementary conditions, and therefore the polynomial used for the trajectory planning should be at least of degree 8 (the position, the velocity and the acceleration when passing through the singularity should be constrained).

In the present study, it will be shown that the degree of the polynomial law should be different, when taking into account the flexibility on the actuating system. Indeed, it will be presented further that the efforts $\tau$ applied on the actuators depend as previously on the position, the velocity and the acceleration of the actuators, but also on the jerk and its first derivative. So, due to the addition of elasticity in the actuated joints, the polynomial used for the trajectory planning should be at least of degree 12 .

In the next section, an example illustrates the obtained results discussed above. This example presents a planar 5R parallel manipulator, which allows obtaining relatively simple symbolic model for demonstrating the expected results by numerical simulations. The results are validated using the ADAMS software.

\section{Illustrative example}

In the planar 5R parallel manipulator, as shown in Fig. 1, the output point is connected to the base by two legs, each of which consists of three revolute joints and two links. In each of the two legs, the revolute joint connected to the base is actuated. Thus, such a manipulator is able to position its output point in a plane.

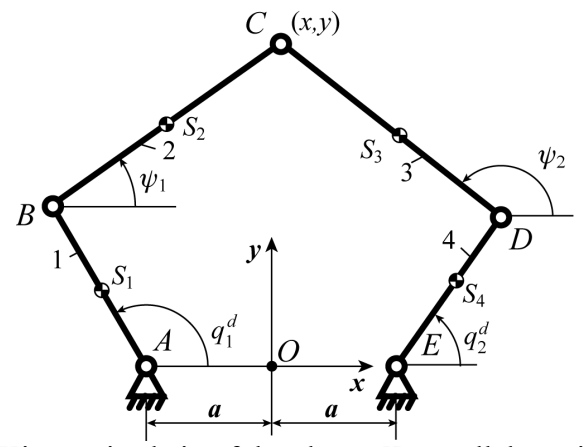

Fig. 1. Kinematic chain of the planar 5R parallel manipulator.

As shown in Fig. 1, the input joints are denoted as $A$ and $E$ with input parameters $q_{1}^{d}$ and $q_{2}^{d}$. The common joint of the two legs is denoted as $C$, which is also the output point with con- trolled parameters $x$ and $y$. A fixed global reference system $\boldsymbol{x} \boldsymbol{O} \boldsymbol{y}$ is located at the center of $A E$ with the $\boldsymbol{y}$-axis normal to $\mathbf{A E}$ and the $\boldsymbol{x}$-axis directed along AE. The lengths of the links $A B, B C, C D$, $D E$ are respectively denoted as $L_{1}, L_{2}, L_{3}$ and $L_{4}$. The positions of the centers of masses $S_{i}$ of links from joint centers $A, B, D$ and $E$ are respectively denoted by dimensionless lengths $r_{1}, r_{2}, r_{3}$ and $r_{4}$, i.e. $A S_{1}=r_{1} L_{1}, B S_{2}=r_{2} L_{2}, D S_{3}=r_{3} L_{3}$ and $E S_{4}=r_{4} L_{4}$.

Actuators 1 and 2 are connected to links 1 and 4 , respectively, via harmonic drive systems which are presented by a model similar to that given in [26]. The position of actuator $i$ is denoted as $q_{i}^{a}$. It is assumed that the actuator $i$ is capable to deliver a couple $\tau_{i}$ to the motor shaft, which is elastically coupled to the link $j$ of the $\operatorname{robot}(i=1$ or $2, j=1$ or 4 ). The flexibility is modeled by a torsion spring with stiffness $k_{i}$. The gear ratio is denoted $n_{i}$. In the following of this paper, $n_{1}=n_{2}=n$ and $k_{1}=k_{2}=k$. $I_{i}^{a}$ is the axial moment of inertia of the motor $i$ plus the Harmonic drive system.

The singularity analysis of this manipulator [29] shows that the Type 2 singularities appear when legs 2 and 3 are parallel (see also Fig. 2 in [1]).

In both cases, the gained degree of freedom is an infinitesimal translation perpendicular to the legs 2 and 3. However, if $L_{2}=L_{3}$, the gained degree of freedom may become a finite rotary motion.

In order to simplify the analytic expressions, we consider that the gravity effects are along the $z$-axis and consequently the input torques are only due to inertia effects. We also admit that there is no friction in the system. To simplify the computation, it is also preferable to replace the masses of moving links by concentrated masses [30, 31]. For a link $i$ with mass $m_{i}$ and its axial moment of inertia $I_{i}$, we have:

$$
\left[\begin{array}{ccc}
1 & 1 & 1 \\
r_{i} & 0 & 1-r_{i} \\
r_{i}^{2} L_{i}^{2} & 0 & \left(1-r_{i}\right)^{2} L_{i}^{2}
\end{array}\right]\left[\begin{array}{c}
m_{i 1} \\
m_{i 2} \\
m_{i 3}
\end{array}\right]=\left[\begin{array}{c}
m_{i} \\
0 \\
I_{i}
\end{array}\right],(i=1,2,3,4)
$$

where $m_{i j}(j=1,2,3)$ are the values of the three point masses placed at the centers of the revolute joints and at the center of masses of the link $i$.

In this case, the potential energy $V$ can be written as:

$$
V=\frac{k}{2}\left(\mathbf{q}_{d}+\mathbf{q}_{a} / n\right)^{T}\left(\mathbf{q}_{d}+\mathbf{q}_{a} / n\right),
$$

where $\mathbf{q}_{d}=\left[q_{1}^{d}, q_{2}^{d}\right]^{T}, \mathbf{q}_{a}=\left[q_{1}^{a}, q_{2}^{a}\right]^{T}$, and the kinetic energy $T$

$$
\begin{aligned}
& T=\frac{1}{2}\left(m_{S 1} \mathbf{V}_{S 1}^{2}+m_{S 2} \mathbf{V}_{S 2}^{2}+m_{S 3} \mathbf{V}_{S 3}^{2}+m_{S 4} \mathbf{V}_{S 4}^{2}+m_{B} \mathbf{V}_{B}^{2}\right. \\
& \left.\quad+m_{C} \mathbf{V}_{C}^{2}+m_{D} \mathbf{V}_{D}^{2}\right)+\frac{1}{2} I_{a} \dot{\mathbf{q}}_{a}^{T} \dot{\mathbf{q}}_{a}
\end{aligned}
$$

where, $\quad m_{S 1}=m_{12}, \quad m_{S 2}=m_{22}, \quad m_{S 3}=m_{32}, \quad m_{S 4}=m_{42}$, $m_{B}=m_{13}+m_{21}, m_{C}=m_{23}+m_{21}, m_{D}=m_{33}+m_{41}$. The terms $m_{i j}$ $(i=1,2,3,4)$ are deduced from the relation (9), $\mathbf{V}_{S i}$ is the vector of the linear velocities of the center of masses $S_{i} ; \mathbf{V}_{B}, \mathbf{V}_{C}$ and $\mathbf{V}_{D}$ are the vectors of the linear velocities of the corresponding axes.

Thus the dynamic model can be obtained from (2):

$$
\begin{gathered}
\mathbf{0}=\mathbf{W}_{\mathbf{b}}+\mathbf{J}_{5 R}^{T} \mathbf{W}_{\mathbf{p}} \\
\tau=I_{a} \ddot{\mathbf{q}}_{a}+\frac{k}{n}\left(\mathbf{q}_{d}+\mathbf{q}_{a} / n\right)
\end{gathered}
$$


taking into account that for examined manipulator:

$$
\mathbf{W}_{\mathbf{b}}=\mathbf{W}_{\mathbf{b}}^{*}+k\left(\mathbf{q}_{d}+\mathbf{q}_{a} / n\right), \mathbf{W}_{\mathbf{b}}^{*}=\mathbf{J}_{\mathbf{B}}^{T} \mathbf{F}_{\mathbf{B}}+\mathbf{J}_{\mathbf{D}}^{T} \mathbf{F}_{\mathbf{D}}
$$

where,

$$
\begin{gathered}
\mathbf{J}_{\mathbf{B}}=\left[\begin{array}{cc}
-L_{1} \sin q_{1} & 0 \\
L_{1} \cos q_{1} & 0
\end{array}\right], \mathbf{J}_{\mathbf{D}}=\left[\begin{array}{cc}
0 & -L_{4} \sin q_{2} \\
0 & L_{4} \cos q_{2}
\end{array}\right], \\
\mathbf{F}_{\mathbf{B}}=m_{B 1} \Gamma_{\mathbf{B}}+m_{C 1} \Gamma_{\mathbf{C}}, \mathbf{F}_{\mathbf{D}}=m_{D 2} \Gamma_{\mathbf{D}}+m_{C 3} \Gamma_{\mathbf{C}} \\
\Gamma_{\mathbf{B}}=L_{1}\left(\ddot{q}_{1}\left[\begin{array}{c}
-\sin q_{1} \\
\cos q_{1}
\end{array}\right]-\dot{q}_{1}^{2}\left[\begin{array}{c}
\cos q_{1} \\
\sin q_{1}
\end{array}\right]\right), \\
\Gamma_{\mathbf{D}}=L_{4}\left(\ddot{q}_{2}\left[\begin{array}{c}
-\sin q_{2} \\
\cos q_{2}
\end{array}\right]-\dot{q}_{2}^{2}\left[\begin{array}{c}
\cos q_{2} \\
\sin q_{2}
\end{array}\right]\right), \Gamma_{\mathbf{C}}=\left[\begin{array}{l}
\ddot{x} \\
\ddot{y}
\end{array}\right] \\
m_{B 1}=m_{S 1} r_{1}^{2}+m_{B}+m_{S 2}\left(1-r_{2}\right)^{2}, m_{C 1}=m_{S 2} r_{2}\left(1-r_{2}\right), \\
m_{C 3}=m_{S 3} r_{3}\left(1-r_{3}\right), m_{D 2}=m_{S 4} r_{4}^{2}+m_{D}+m_{S 3}\left(1-r_{3}\right)^{2} .
\end{gathered}
$$

The term $\mathbf{W}_{\mathbf{p}}$ is given by:

$$
\begin{gathered}
\mathbf{W}_{\mathbf{p}}=m_{C 1} \boldsymbol{\Gamma}_{\mathbf{B}}+m_{C 2} \Gamma_{\mathbf{C}}+m_{C 3} \Gamma_{\mathbf{D}}, \\
m_{C 2}=m_{S 2} r_{2}^{2}+m_{C}+m_{S 3} r_{3}^{2} .
\end{gathered}
$$

and the Jacobian matrix $\mathbf{J}_{5 R}$ by:

$$
\mathbf{J}_{5 R}=\mathbf{A}_{5 R}^{-1} \mathbf{B}_{5 R},
$$

where,

$$
\begin{array}{r}
\mathbf{A}_{5 R}=\left[\begin{array}{ll}
a_{11} & a_{12} \\
a_{21} & a_{22}
\end{array}\right]=2\left[\begin{array}{cc}
x-L_{1} \cos q_{1}+a & y-L_{1} \sin q_{1} \\
x-L_{4} \cos q_{2}-a & y-L_{4} \sin q_{2}
\end{array}\right], \\
\mathbf{B}_{5 R}=-\left[\begin{array}{cc}
L_{1}\left(a_{11} \sin q_{1}-a_{12} \cos q_{1}\right) & 0 \\
0 & L_{4}\left(a_{21} \sin q_{2}-a_{22} \cos q_{2}\right)
\end{array}\right] .
\end{array}
$$

For a given trajectory $\mathbf{q}_{d}$, the vector of the positions of the actuators $\mathbf{q}_{a}$ can be deduced from (12a), as well as the acceleration of the actuators $\ddot{\mathbf{q}}_{a}$ :

$$
\begin{gathered}
\mathbf{q}_{a}=-\frac{n}{k}\left(\mathbf{W}_{\mathbf{b}}^{*}+\mathbf{J}_{5 R}^{T} \mathbf{W}_{\mathbf{p}}\right)-n \mathbf{q}_{d} \\
\ddot{\mathbf{q}}_{a}=-\frac{n}{k} \frac{d^{2}}{d t^{2}}\left(\mathbf{W}_{\mathbf{b}}^{*}+\mathbf{J}_{5 R}^{T} \mathbf{W}_{\mathbf{p}}\right)-n \ddot{\mathbf{q}}_{d}
\end{gathered}
$$

Introduction Eq. (24) into Eq. (12b), one can deduce the vector of actuator torques $\tau$ :

$$
\tau=-I_{a}\left(\frac{n}{k} \frac{d^{2}}{d t^{2}}\left(\mathbf{W}_{\mathbf{b}}^{*}+\mathbf{J}_{5 R}^{T} \mathbf{W}_{\mathbf{p}}\right)+n \ddot{\mathbf{q}}_{d}\right)-\frac{1}{n}\left(\mathbf{W}_{\mathbf{b}}^{*}+\mathbf{J}_{5 R}^{T} \mathbf{W}_{\mathbf{p}}\right) .
$$

Analyzing this expression, it could be observed that, as terms $\mathbf{W}_{\mathbf{b}}^{*}$ and $\mathbf{W}_{\mathbf{p}}$ depend on the position, velocity and acceleration of the input links, the input torques depend not only on these parameters, but also on the jerk and its first derivative. Therefore, on the contrary of rigid manipulators for which, in order to avoid discontinuities in the input torques, a fifth-degree polynomial is sufficient as a control law when the end-effector is not in singular configuration, for non rigid robots, the degree of the polynomial should be increased (indeed, it should be at least a ninth-degree polynomial).

In order to avoid infinite values of the input torques when crossing a Type 2 singularity, Eq. (7) has to be satisfied. From matrix $\mathbf{A}_{5 R}$, one can find that the twist of the infinitesimal displacement in the singularity can be written under the form:

$$
\mathbf{t}_{\mathbf{s}}=\left[-\sin \psi_{1}, \cos \psi_{1}\right]^{T}
$$

Thus, the examined manipulator can pass through the given singular positions if the wrench $\mathbf{W}_{\mathbf{p}}$ determined by (19) is orthogonal to the direction of the uncontrollable motion $\mathbf{t}_{\mathbf{s}}$ described by (26).

Let us now consider the motion planning, which makes it possible to satisfy this condition. For this purpose the following parameters of manipulator's links are specified: $L_{1}=L_{2}=L_{3}=L_{4}=$ $0.25 \mathrm{~m} ; r_{1}=r_{2}=r_{3}=r_{4}=0.5 ; a=0.2 \mathrm{~m} ; m_{1}=m_{4}=2.81 \mathrm{~kg} ; I_{1}=$ $I_{4}=0.02 \mathrm{~kg} \cdot \mathrm{m}^{2} ; m_{2}=m_{3}=1.41 \mathrm{~kg} ; I_{2}=I_{3}=0.01 \mathrm{~kg} \cdot \mathrm{m}^{2} ; I_{a}=$ $0.067 \mathrm{~kg} / \mathrm{m}^{2} ; k=250 \mathrm{Nm} / \mathrm{rad} ; n=50$.

With regard to the prescribed trajectory generation, the point $C$ should reproduce a motion along a straight line between the initial position $C_{0}\left(x_{0}, y_{0}\right)=C_{0}(0.1,0.345)$ and the final point $C_{f}\left(x_{f}\right.$, $\left.y_{f}\right)=C_{f}(-0.1,0.145)$ in $t_{f}=2 \mathrm{~s}$ (Fig. 2).

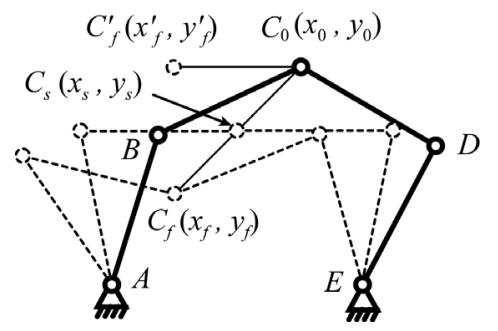

Fig. 2. Initial, singular and final positions of the planar 5R parallel manipulator.

Thus, the given trajectory can be expressed as follows:

$$
\mathbf{x}=\left[\begin{array}{l}
x(t) \\
y(t)
\end{array}\right]=\left[\begin{array}{l}
x_{0}+s(t)\left(x_{f}-x_{0}\right) \\
y_{0}+s(t)\left(y_{f}-y_{0}\right)
\end{array}\right]
$$

However, the manipulator will pass by a Type 2 singular position at point $C_{s}\left(x_{s}, y_{s}\right)=C_{s}(0,0.245)$ (Fig. 2).

Developing the condition for passing through the singular position (7) for the planar 5R parallel manipulator at point $C_{s}$, we obtain:

$$
m_{C 1} L_{1}\left(248 \dot{x}^{2}-48 \dot{y}^{2}\right)-3 \sqrt{6} m_{C 2} \ddot{y}=0
$$

Then, taking into account that the velocity and the acceleration of the end-effector in initial and final positions are equal to zero, the following thirteen boundary conditions are found:

$$
\begin{gathered}
s\left(t_{0}\right)=0, \\
s\left(t_{f}\right)=1, \\
s\left(t_{s}=1 \mathrm{~s}\right)=0.5, \\
\dot{s}\left(t_{0}\right)=0, \\
\dot{s}\left(t_{f}\right)=0, \\
\dot{s}\left(t_{s}\right)=\dot{y}_{s} /\left(y_{f}-y_{0}\right)=\dot{x}_{s} /\left(x_{f}-x_{0}\right)=1, \\
\ddot{s}\left(t_{0}\right)=\ddot{s}_{0}=0, \\
\ddot{s}\left(t_{f}\right)=\ddot{s}_{f}=0, \\
\ddot{s}\left(t_{s}\right)=\ddot{s}_{s}=m_{C 1} L_{1}\left(248 \dot{x}_{s}^{2}-48 \dot{y}_{s}^{2}\right) /\left(3\left(y_{f}-y_{0}\right) \sqrt{6} m_{C 2}\right), \\
\dddot{s}\left(t_{0}\right)=\dddot{s}_{0}=0, \\
\dddot{s}\left(t_{f}\right)=\dddot{s}_{f}=0, \\
\frac{d}{d t} \dddot{s}\left(t_{0}\right)=s_{0}^{(4)}=0, \\
\frac{d}{d t} \dddot{s}\left(t_{f}\right)=s_{f}^{(4)}=0 .
\end{gathered}
$$


Table 1. Variation of the input torques as a function of the polynomial law used for the trajectory.

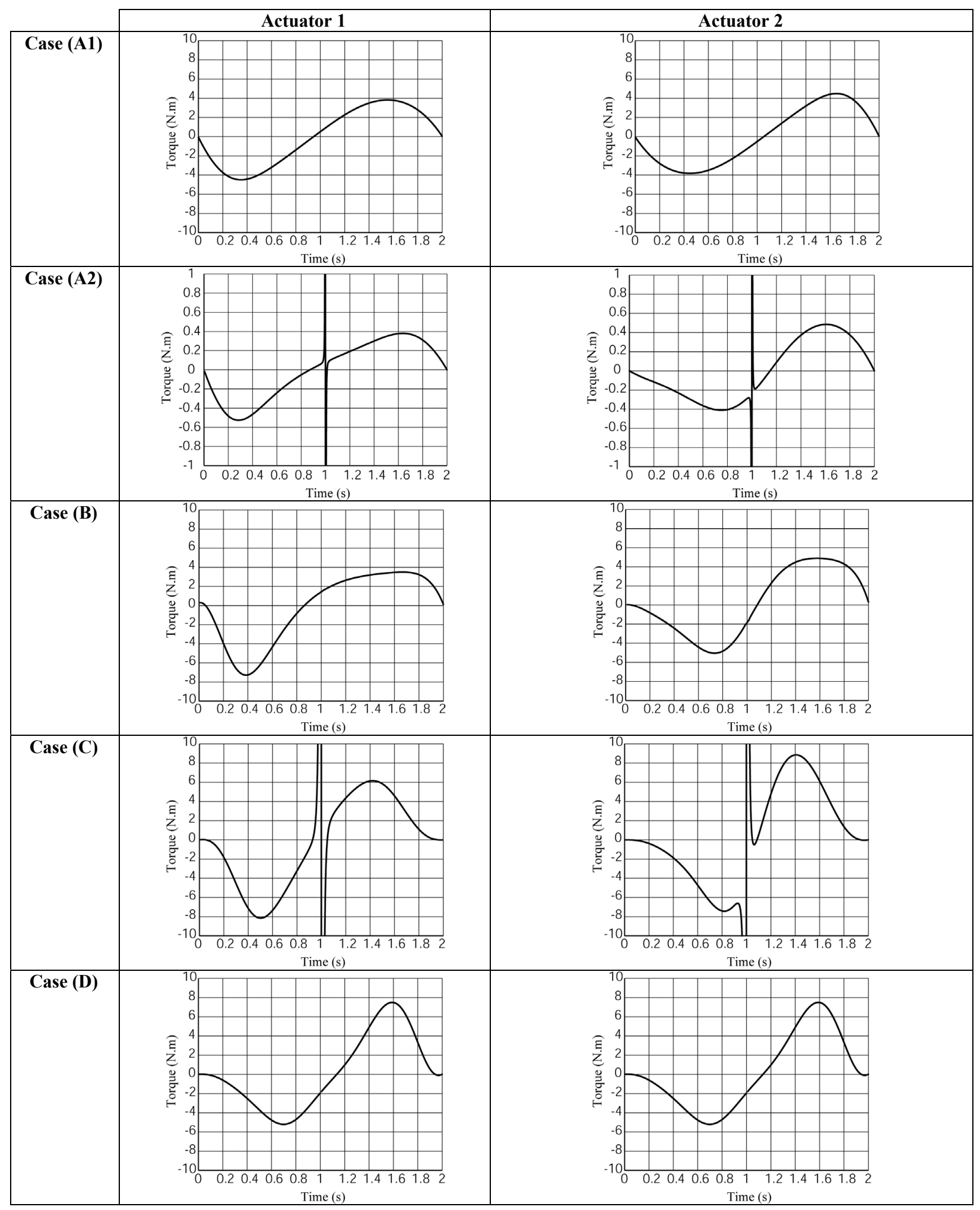


From (28)-(41), the following twelfth order polynomial trajectory planning is found:

$$
\begin{aligned}
s(t)= & 7.28 t^{5}-14.22 t^{6}+6.80 t^{7}+6.41 t^{8}-9.77 t^{9} \\
& +5.15 t^{10}-1.28 t^{11}+0.13 t^{12}
\end{aligned}
$$

Thus the generation of the motion by the obtained twelfth order polynomial makes it possible to pass through the singularity without perturbation and the input torques remain in the limits of finite values.

In order to compare the different cases of trajectory planning, in table 1 are given the values of the input torques obtained using the software ADAMS for the following numerical simulations:

A1: a trajectory between points $C_{0}$ and $C_{f}^{\prime}\left(x_{f}^{\prime}, y_{f}^{\prime}\right)=C_{f}^{\prime}(-$ $0.1,0.345$ ) (Fig. 2) without meeting any singularity. For such a case, the following fifth order polynomial law is used $s(t)=1.25 t^{3}-0.9375 t^{4}+0.1875 t^{5}$ for the trajectory planning out of the singular zone of the rigid-link manipulator without taking into account the flexibility in the actuated joints. In this case the values of the input torques are finite.

A2: the fifth order polynomial law $s(t)=1.25 t^{3}-0.9375 t^{4}+0.1875 t^{5}$ for the trajectory planning between $C_{0}$ and $C_{f}$ inside the singular zone for the rigid-link manipulator without taking into account the flexibility in the actuated joints. In this case the values of the input torques close to the singular positions tend to infinity.

B: the eight order polynomial law $s(t)=-0.25851 t^{3}+$ $3.84228 t^{4}-5.72792 t^{5}+3.58909 t^{6}-1.07101 t^{7}+$ $0.12606 t^{8}$ for the trajectory planning of the rigid-link manipulator without flexibility in the actuated joints inside the singular zone. The obtained results show that the values of the input torques are finite.

$\mathrm{C}$ : the ninth order polynomial law $s(t)=3.94 t^{5}-6.56 t^{6}+4.22 t^{7}-1.23 t^{8}+0.14 t^{9} \quad$ for the trajectory planning of the rigid-link flexible-joint manipulator inside the singular zone. The numerical simulation shows that the values of the input torques close to the singular positions tend to infinity.

D: the twelfth order polynomial law (42) for the trajectory planning of the rigid-link flexible-joint manipulator inside of the singular zone. The values of the input torques are finite and there are no discontinuities.

Thus, the numerical simulations show that the obtained optimal dynamic conditions assume the passing of the rigid-link flexiblejoint manipulator through the singular position.

\section{Conclusion}

At a singular configuration, in the case of an arbitrary generation of forces, a manipulator may not reproduce stable motion with prescribed trajectory. Nevertheless it is approved that there are several motion planning techniques, which allow passing through these singular zones. These approaches are simulated by numerical examples and illustrated on several parallel structures. However, in these studies much more attention was focused only on control aspects of this problem and little attention has been paid to the dynamic interpretation, which is a crucial factor for governing the behavior of parallel manipulators at the singular zones.

In our previous work [1], the dynamic properties of parallel manipulators in the presence of Type 2 singularity have been studied. It was shown that any parallel manipulator can pass through the singular positions without perturbation of motion if the wrench applied on the end-effector by the legs and external efforts of the manipulator are orthogonal to the twist along the direction of the uncontrollable motion. This condition was applied to the rigid-link manipulators without clearance or flexibility in the joints. The obtained results showed that the planning of motion for assuming the optimal force generation can be carried out by a eight order polynomial law.

In the present paper the rigid-link flexible-joint manipulators have been studied. It was shown that the degree of the polynomial law should be different, when the flexibility of actuated joints is introduced into conditions of the optimal force generation in the presence of singularity. The obtained results disclosed that the planning of motion for assuming the optimal force generation in the rigid-link flexible-joint manipulators must be carried out by a twelfth order polynomial law. The suggested technique was illustrated by an example, which presents $5 \mathrm{R}$ planar parallel manipulator with flexible joints. The numerical simulations carried out using the software ADAMS validated the obtained theoretical results.

\section{References}

[1] Briot, S., and Arakelian, V., 2008, "Optimal Force Generation in Parallel Manipulators for Passing through the Singular Positions," International Journal of Robotics Research, Vol. 27, No. 8, pp.967-983.

[2] Gosselin, C.M., Angeles, J., 1990, "Singularity Analysis of Closed-Loop Kinematic Chains," IEEE Transactions on Robotics and Automatics, Vol.6, No.3, pp.281-290.

[3] Zlatanov, D., Fenton, R.G., Benhabib, B., 1994, "Singularity Analysis of Mechanisms and Robots via a VelocityEquation Model of the Instantaneous Kinematics," Proceedings of the 1994 IEEE International Conference on Robotics and Automation, Vol. 2, pp.980-991.

[4] Zlatanov, D., Bonev, I.A., Gosselin, C. M., 2002, "Constraint Singularities of Parallel Mechanisms," IEEE International Conference on Robotics and Automation (ICRA 2002), Washington, D.C., USA, May 11-15.

[5] Hunt, K.H., 1987, "Special Configurations of Robot-Arms via Screw Theory," Robotica, Vol. 5, pp. 17-22.

[6] Merlet, J.-P., 1989, "Singular Configurations of Parallel Manipulators and Grassmann Geometry," The International Journal of Robotics Research, Vol. 8, No. 5, pp.45-56.

[7] Bonev, I.A., Zlatanov, D., Gosselin, C.M., 2003, "Singularity Analysis of 3-DOF Planar Parallel Mechanisms via Screw Theory," ASME Journal of Mechanical Design, Vol. 125 , No. 3, pp.573-581.

[8] Briot, S. and Arakelian, V., 2007, "Singularity Analysis of PAMINSA Manipulator." The 12th World Congress in Mechanism and Machine Science, 18-21 June 2007, Besançon, France.

[9] Gosselin, C.M., 1992, "The Optimum Design of Robotic Manipulators using Dexterity Indices," Robotics and Autonomous Systems, Vol. 9, No. 4, pp.213-226. 
[10] Merlet, J.-P., 2006, “Jacobian, Manipulability, Condition Number, and Accuracy of Parallel Robots," ASME Journal of Mechanical Design, Vol. 128, No. 1, pp.199-206.

[11] Angeles, J., 2007, "Fundamentals of Robotic Mechanical Systems: Theory, Methods, and Algorithms," 3rd edition, Springer, New York.

[12] Alba-Gomez, O., Wenger, P., and Pamanes, A., 2005, September 24-28, "Consistent Kinetostatic Indices for Planar 3DOF Parallel Manipulators, Application to the Optimal Kinematic Inversion," Proc. ASME 2005 IDETC/CIE Conference, Long Beach, California.

[13] Arakelian, V., Briot, S., and Glazunov, V., 2008, "Increase of Singularity-Free Zones in the Workspace of Parallel Manipulators using Mechanisms of Variable Structure," Mechanism and Machine Theory, Vol. 43, No. 9, pp. 11291140.

[14] Hubert, J and Merlet, J.-P., 2008, "Singularity Analysis through Static Analysis," Advances in Robot Kinematics, Springer, pp.13-20.

[15] Alvan, K., and Slousch, A., 2003, "On the Control of the Spatial Parallel Manipulators with Several Degrees of Freedom," Mechanism and Machine Theory, Saint-Petersburg, No. 1, pp. 63-69.

[16] Dasgupta, B., and Mruthyunjaya, T., 1998, "Force Redundancy in Parallel Manipulators: Theoretical and Practical Issues," Mechanism and Machine Theory, Vol. 33, No. 6, pp.724-742.

[17] Glazunov, V., Kraynev, A., Bykov, R., Rashoyan, G., and Novikova, N., 2004, "Parallel Manipulator Control While Intersecting Singular Zones," Proc. 15th Symposium on Theory and Practice of Robots and Manipulators (RoManSy) CISM-IFToMM, Montreal.

[18] Kotlarski, J., Do Thanh, T., Abdellatif., H., and Heimann, B., 2008 "Singularity Avoidance of a Kinematically Redundant Parallel Robot by a Constrained Optimization of the Actuation Forces," In the Proceedings of the Seventeenth CISM-IFToMM Symposium RoManSy, pp.435-442.

[19] Bhattacharya, S., Hatwal, H., and Ghosh, A., 1998, "Comparison of an Exact and Approximate Method of Singularity Avoidance in Platform Type Parallel Manipulators," Mechanism and Machine Theory, Vol. 33, No. 7, pp. 965974.

[20] Dasgupta, B., and Mruthyunjaya, T., 1998, "Singularity-Free Path Planning for the Steward Platform Manipulator," Mechanism and Machine Theory, Vol. 33, No. 6, pp. 715725.

[21] Hesselbach, J., Wrege, J., Raatz A., and Becker, O., 2004, "Aspects on the design of high precision parallel robots," Assembly Automation, Vol. 24, No. 1, pp. 49-57.

[22] Kemal Ider, S., 2005, "Inverse Dynamics of Parallel Manipulators in the Presence of Drive Singularities," Mechanism and Machine Theory, Vol. 40, pp. 33-44.

[23] Kevin Jui, C.K., and Sun, Q., 2005, "Path Tracking of Parallel Manipulators in the Presence of Force Singularity," Transactions of the ASME. Journal of Dynamic Systems, Measurement and Control, Vol. 127, pp. 550-563.

[24] Nenchev, D.N., Bhattacharya, S., and Uchiyama, M., 1997, "Dynamic analysis of parallel manipulators under the singularity-consistent parameterization," Robotica, Vol. 15, No. 4, pp. 375-384.
[25] Perng, M.H., and Hsiao, L., 1999, "Inverse Kinematic Solutions for a Fully Parallel Robot with Singularity Robustness," The International Journal of Robotics Research, Vol. 18, No. 6, pp. 575-583.

[26] Spong, M.W., Khorasani, K., and Kokotovic, P.V., 1987, "An Integral Manifold Approach to the Feedback Control of Flexible Joint Robots," IEEE Journal of Robotics and Automation, Vol. 3, No. 4, pp. 291-300.

[27] Khalil, W., and Guégan, S., 2002, May 11-15, “A Novel Solution for the Dynamic Modeling of Gough-Stewart Manipulators," Proc. IEEE International Conference on Robotics and Automation, Washington DC., USA.

[28] Merlet, J.-P., 2006, "Parallel Robots," Springer, 2nd edition.

[29] Liu, X.-J., Wang, J., and Pritschow, G., 2006, "Kinematics, Singularity and Workspace of Planar 5R Symmetrical Parallel Mechanism," Mechanism and Machine Theory, Vol. 41, No. 2, pp. 119-144.

[30] Seyferth, W., 1974, "Massenersatz duch Punktmassen in Räumlichen Getrieben," Mechanism and Machine Theory, Vol. 9, pp. 49-59.

[31] Wu, Y., and Gosselin, C.M., 2007, "On the Dynamic Balancing of Multi-DOF Parallel Mechanisms with Multiple Legs," Transaction of the ASME. Journal of Mechanical Design, Vol. 129, No. 2, pp. 234-238. 